36

\section{Blood flow velocity in medial cerebral artery during observation of own arm movement in a mirror}

Béné Raphael, Arijana Lovrenčić-Huzjan, Ažman Dražen, Strineka

Maja, Budišić Mislav, Vuković Vlasta, Rastovčan Boško

\& Demarin Vida

University Department of Neurology, Sestre Milosrdnice University

Hospital, Reference Center for Neurovascular Disorders of Republic of

Croatia, Reference Center for Headache of Republic of Croatia, Zagreb,

Croatia, Vinogradska Cesta 29

E-mail: Raphaelbene.hr@gmail.com

Introduction: Mirror illusion consist in the fact that, standing in front of a mirror put in a sagittal plane, with our head on one side and one arm stretched forward, we can see one side of our body reflected as if it were the other side, by mirror visual feedback.

The aim of this study was to monitor blood flow changes in medial cerebral artery (MCA) by means of Transcranial Doppler (TCD) in individuals during motor tasks as well as tasks using mirror visual feedback.

Subjects and methods: Eight young healthy volunteers (four male and four female) participated in this study. TCD recording of MCA was done during each task consisting of various motor and visuo-motor activities using mirror illusion. Both MCA mean blow flow velocity (MBFV) were measured while individuals seated in a comfortable chair. The obtained MCA MBFV are presented as baseline values

Results: During the illusion of motoric hand activation, when the subject is making right hand flexions and watching its reflexion in the mirror, while the left hand is immobile, increase of mean blood flow velocity of contralateral MCA was observed (task $3+4.5 \%$ than in baseline values, $P=0.017$ ).

Furthermore, when the subject made left hand flexions while watching the reflection of the immobile right hand in the mirror, there was increase of MBFV in right MCA $(+5.6 \%$ than in baseline values $P=0.044)$, more pronounced than during the illusion of motoric hand activation (task 3 ) and less than during direct vision of hand flexion (task $2+6.3 \%$ than in baseline values $P=0.005$ ).

Conclusion: Our data showed that visual illusion of action, as well as direct action observation can increase mean blood flow value in MCA, which brings forward the possible usage of mirror illusion as a tool for motoric neurorehabilitation.

Key words: Transcranial Doppler, mirror illusion, medial cerebral artery

\section{7}

\section{Dissection of the craniocervical arteries}

Marija Bošnjak-Pašić1, Vesna Vargek-Solter ${ }^{1}$, Melita Uremović ${ }^{3}$, Branka Bošnjak ${ }^{2}$, Renata Budić ${ }^{4}$, Darko Solter ${ }^{1} \&$ Vida Demarin $^{1}$ ${ }^{1}$ University Department of Neurology, Sestre Milosrdnice University Hospital, Zagreb, Croatia, ${ }^{2}$ University Department of Psychiatry, Alcoholism and Other Dependences, Sestre Milosrdnice University Hospital, Zagreb, Croatia, Insurance Company, Zagreb, Croatia, ${ }^{4}$ Special Hospital for Rehabilitation, Krapinske Toplice

Once considered rare, dissection of the internal carotid artery or vertebral artery is an increasingly recognized entity. Craniocervical arterial dissection is an uncommon cause of stroke in the general population, but is relatively common in patients below the age of 40 . The early clinical manifestations are often subtle, however, permanent neurologic disability and death can result if the diagnosis is delayed. The present report describes eight patients (four men and four women) with dissection of the craniocervical arteries. Patient history was taken and clinical neurologic examinations were performed immediately upon admission. Diagnostic procedures included ultrasound (CDFI and TCD) and radiologic (computed tomography and digital subtraction angiography) examinations. The leading symptoms were focal neurologic deficits, and headache and neck ache. Ultrasound findings (CDFI) were positive for vessel dissection in seven $(87.5 \%)$ patients, and DSA was consistent with dissection in five $(62.5 \%)$ patients and negative in one patient, whereas in two patients this examination was not performed due to the known allergy to contrast medium. Five $(62.5 \%)$ patients were treated with anticoagulants, one with platelet aggregation suppressants, and two patients were operated on. Six $(75.0 \%)$ patients showed partial post-therapeutic recovery of neurologic deficits and improvement of ultrasound findings of dissected arteries. One patient developed a stroke postoperatively, with deterioration of the motor deficit, and one patient was readmitted 3 months later for a newly developed stroke and died soon thereafter. It is concluded that early detection of craniocervical arterial dissection is important to minimize the morbidity and mortality associated with this condition.

\section{8 \\ Response to music in patients with acute ishaemic stroke}

S. Antić, V. Vargek Solter, A. Lovrenčić-Huzjan, V. Supanc, S. Morović, M. J. Jurašić \& V. Demarin

University Department of Neurology, University Hospital Sestre

Milosrdnice, Vinogradska Cesta 29, Zagreb, Croatia

E-mail: sonja.antic@gmail.com

Background: Auditory stimulation increases mean blood flow velocity (MBFV) in the middle cerebral artery (MCA). Our aim was to monitor such changes in the affected MCA in patients with acute ischemic stroke (AIS) and to compare them with healthy individuals.

Methods: The study included 113 examinees (52 non-thrombolysed patients admitted to the hospital within 24 hours of AIS onset, and 61 healthy individuals). A baseline MCA MBFV was recorded by means of transcranial Doppler (TCD). In stroke patients only the affected MCA was insonated while in healthy controls both MCAs were recorded. MCA MBFV was monitored during listening to relaxing music for 2 minutes. The first response of MBFV increase was measured as time (Tmax) and percentage of change in amplitude (Amax). Pearson Chi-Square test was used for intergroup comparison.

Results: At baseline, stroke patients had lower MCA MBFV than healthy individuals $(P<0.01)$. In both groups there was an increase in MBFV compared to baseline values as a reaction to the music. Tmax in stroke group $(15.87 \pm 7.72)$ was significantly longer $(P<0.01)$ than Tmax in control group $(9.34 \pm 6.16)$. There was no statistically significant difference in Amax between the two groups.

Conclusion: Even though stroke patients with MCA branch occlusion have impaired blood flow in the affected MCA, music is still an effective auditory stimulus. However, their time of reaction to the music was prolonged when compared to healthy controls.

\section{9}

\section{Whiplash injury - a medicolegal issue}

Marija Bošnjak-Pašić1, Melita Uremović2 ${ }^{2}$ Branka Vidrih ${ }^{3}$, Vesna Vargek-Solter ${ }^{1}$, Marijana Lisak ${ }^{1} \&$ Vida Demarin ${ }^{1}$

${ }^{1}$ University Department of Neurology, Sestre Milosrdnice University Hospital, ${ }^{2}$ Zagreb Insurance Company, ${ }^{3}$ University Department of Psychiatry, Sestre Milosrdnice University Hospital, Zagreb, Croatia

Whiplash injury is the most common injury sustained in traffic accidents. On exposure to different forces, multiple deck injuries may occur. Following the injury, many patients suffer from 